\title{
NOVEDADES EN EL GÉNERO GAYA (MALVACEAE)
}

\author{
ANTONIO KRAPOVICKAS ${ }^{1}$
}

Summary: Krapovickas, A. 2012. Novelties in the genus Gaya (Malvaceae). Bonplandia 21(1): 71-76.

The history of de problematical name Sida sylvatica Cav. is analyzed and the new combination Gaya sylvatica (Cav.) Krapov. is proposed. The new species G. Kelleri, from Misiones, Argentina is described.

Key words: Malvaceae, Gaya, taxonomy, Argentina, Peru.

Resumen: Krapovickas, A. 2012. Novedades en el género Gaya (Malvaceae). Bonplandia 21(1): 71-76.

La historia del nombre problemático Sida sylvatica Cav. es abordado y se propone la nueva combinación Gaya sylvatica (Cav.) Krapov. Se describe la nueva especie G. Kelleri de Misiones, Argentina.

Palabras clave: Malvaceae, Gaya, taxonomía, Argentina, Perú.

\section{Introducción}

El género Gaya H.B.K. tiene una posición aislada en la familia de las Malváceas (s.str.) por la presencia de una lengüeta o endoglosa muy ornamentada en la base de sus mericarpios, la que puede ser muy reducida o faltar completamente en algunas de sus especies. Sin embargo hay una cantidad de caracteres que sirven para definir este género (Krapovickas \& al. 1974; Hanks \& Fryxell, 1979; Krapovickas 1996).

En esta oportunidad se presentan dos novedades que pertenecen al grupo de endoglosa reducida o ausente: sección Microlophia K.Schum. y una clave para reconocer las especies pertenecientes a esta sección.

\section{Sección Gaya}

Lectotypus: Gaya hermanioides H.B.K. (Fryxell, 1992: 36).

Endoglosa con márgenes dentados, la cual sobrepasa y envuelve a la semilla.

\section{Sección Microlophia K. Schum.}

Lectotypus, aquí designado: Gaya pilosa K.Schum. in Mart. Fl.bras. 12(3): 355, 1891.

Endoglosa incipiente, menor que la semilla o ausente.

\footnotetext{
${ }^{1}$ Instituto de Botánica del Nordeste, Casilla de Correo 209, 3400 Corrientes, Argentina.
} 


\section{Las especies de Gaya sect. Microlophia se pueden identificar por medio de la siguiente clave}

A. Mericarpos con endoglosa muy pequeña.

B. Flores solitarias o de a dos axilares.

C. Flores solitarias, axilares.

D. Cáliz piloso, pelos 0,5-2 mm long. Tallo velutino, con pelos estrellados y glandulares, y pelos simples 1-2 mm long. Mericarpos 23-25 (6 x 4-5 mm). Pétalos $15 \mathrm{~mm}$ long. $2 \mathrm{n}=12$. Argentina, Bolivia, Brasil, Paraguay.

G. pilosa K.Schum.

D'.Cáliz con pelos menores de 0,3 mm long. Tallo velutino. Mericarpos 12-17.

E. Cáliz acrescente, con 5 gibas, notables en el fruto. Pétalos 15 mm long. Mericarpos 13-17 $2 n=12$. Brasil.

G. Guerkeana K.Schum.

E'.Cáliz no acrescente, liso o con costillas sobresalientes en el fruto (G. Kelleri).

F. Endoglosa lisa, envuelve la semilla pero no la sobrepasa. Pétalos 18-22 mm long. Mericarpos 16. Brasil, Bahia, M. Gerais.

G. aurea A.St.Hil.

F'. Endoglosa muy pequeña, lisa, apenas incipiente. Corola $25 \mathrm{~mm}$ long. Mericarpos 12-15. Brasil, Pará.

G. scopulorum Krapov.

C'. Flores de a dos por axila. Mericarpos 30-36. Perú, Huánuco.

G. sylvatica (Cav.) Krapov.

B'. Flores en racimos terminales, áfilos. Corola $10 \mathrm{~mm}$ long. Mericarpos 16-17. 2n=12. Argentina, Misiones.

G. Kelleri Krapov.

A'. Mericarpos sin endoglosa, dehiscentes. Flores solitarias, axilares.

G. Mericarpos 25-39 (14x 10 mm). Tallo velutino y con largas setas $2 \mathrm{~mm}$ long. 2n=12. Brasil, Bahía.

G. monosperma (K.Schum.) Krapov.

G'.Mericarpos 12 (5 x6 mm). Tallo velloso, con pelos hasta 0,5 mm long. Venezuela, Mérida.

G. meridensis Krapov.

Gaya sylvatica (Cav.) Krapov., comb. nov.

Fig. 1

Sida sylvatica Cav., Diss. 2: 56-57. 1786. Typus: "in sylvis prope flumen Maragnon in Perú. V. S.communicatum a D.Dombey". Holotypus: in sylvis prope Maragnon, Perú [dep. Huánuco], dadas por el Sr. Dombey [letra de Cavanilles] (MA 29760, foto F 29760!).

Cav. Diss. 5: 276, tab.133, fig. 2. 1788. [la misma figura se reproduce en Lamarck, Tabl.encycl. 3, tab. 578 fig. 2. 1794].

Abutilon sylvaticum (Cav.) K.Presl. Reliq. haenk. 2: 114.1835 .
Abutilon sylvaticum (Cav.) K.Schum. in Martius, Fl. bras. 12(3): 418-419. 1891.

Cavanilles (1786: 56) describe Sida sylvatica sobre material cedido por Dombey, procedente de las nacientes del río Marañón, en el departamento de Huánuco (ca $10^{\circ} \mathrm{S} 76-$ $77^{\circ} \mathrm{W}$ ), Perú, donde Dombey junto con Ruiz y Pavón estuvieron durante su famoso viaje a Perú y Chile (1777-1788).

De Candolle (1824: 466) ubica a Sida sylvatica en la sección II. Abutiloides, que abarca a los géneros Gaya y Bastardia de Humbold, Bonpland y Kunth. 
El criterio de De Candolle es seguido por G.Don (1831, 1: 497), quién acepta Sida sylvatica Cav. En pág. 500 eleva Sida secc. Abutilon DC, con carpelos polispermos, al rango de género. En pág. 503 al tratar Abutilon geminiflorum H.B.K. compara esta especie con "A.sylvaticum", que tiene una sola semilla en cada carpelo, sin referencia al autor.

Garcke (1893: 489) sostiene que se trata de una nueva combinación: Abutilon sylvaticum (Cav.) G.Don, a pesar de lo ambiguo de la cita de Don, sin dar ninguna garantía de su relación con la especie de Cavanilles.

En 1835 Presl al describir Abutilon dianthum K.Presl, dice "affine A.sylvatico (Sida sylvatica Cav.)", estableciendo formalmente la nueva combinación.

K.Schumann (1891: 418-419) publica Abutilon silvaticum (Cav.) K.Schum. y señala que Cavanilles se debe haber equivocado al atribuir a Sida sylvatica "Capsulae 30-36 compressae, muticae, monospermae". Según la descripción de K.Schumann, el material de Perú que él estudió proviene del departamento de Huánuco, tiene "ovarium 9-carpidiatum" y "loculis 7-ovulatis", que actualmente se identifica como A.dianthum K.Presl (Fryxell, 1992: 10, Fuertes \& Fryxell, 1993).

La lámina de Cavanilles es bien clara, ilustra el gineceo con 36 estigmas en cabezuela, lo cual coincidiría con "capsulae 30-36", por lo cual se diferencia fácilmente de A.dianthum. Lo que tienen en común estas dos especies son las dos flores en cada axila, agrupadas hacia el ápice de las ramas y con pedúnculos hasta 12 $\mathrm{cm}$ long. (dianthum $=$ dos flores).

Fuertes \& Fryxell (1993) consideran que Sida sylvatica Cav. "is a problematic name". Ellos vieron el holotipo (MA-Cav s.n.) y un aparente isotipo en el herbario general: "Sida - 1780 - Cochexo (MA 265804) y no pueden interpretar la localidad Cochexo, pero se parece mucho a Cuchero, localidad visitada por Ruiz, Pavón y Dombey.

La posición genérica de Sida sylvática es un problema. Todos los autores la tratan como Abution sylvaticum (Cav.) K.Schum., pero no puede serlo por sus carpelos "monospermae" (Fuertes \& Fryxell, 1993).

En mi opinión la mejor posibilidad es el género Gaya H.B.K. La cantidad de carpelos (30-36)
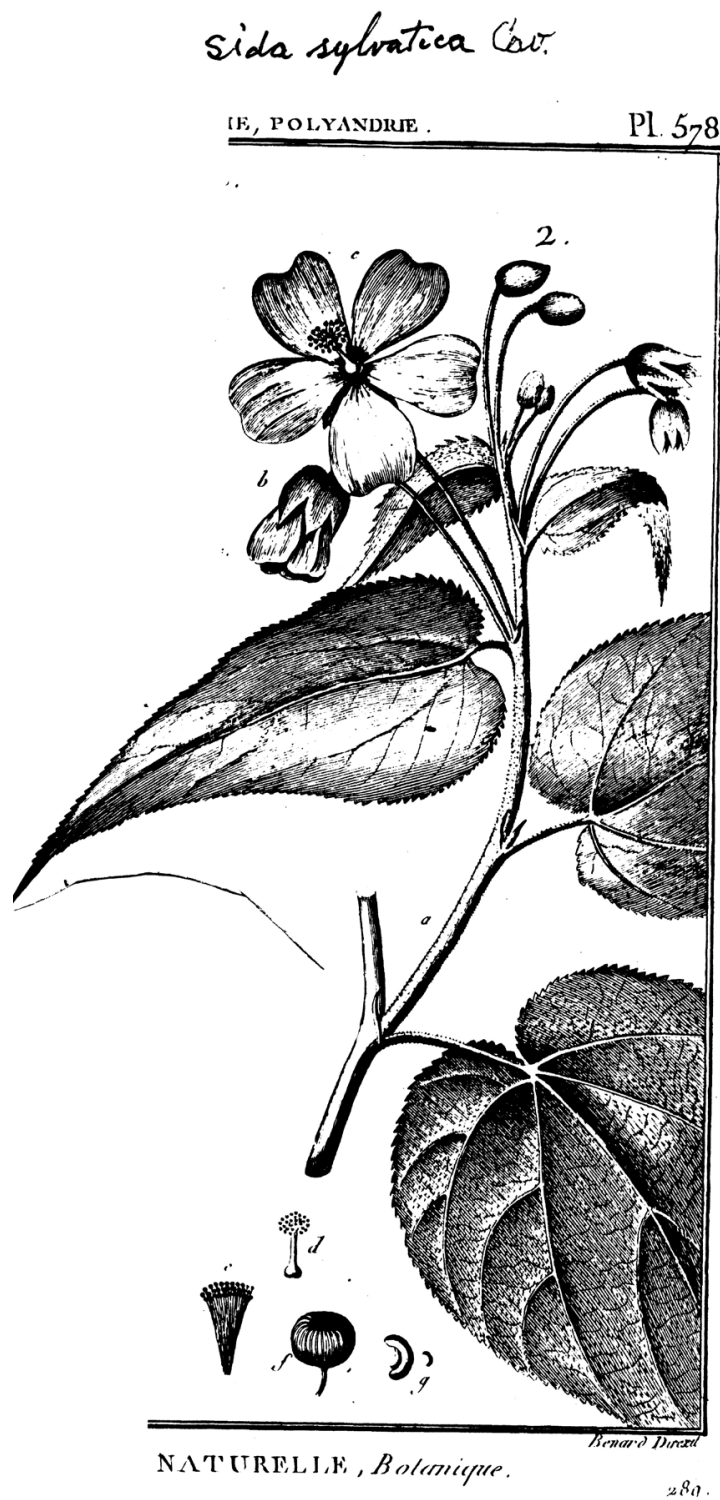

tomads de Cow. diss. 5 tal. 133 f. 2

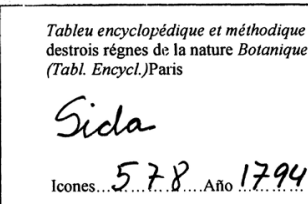

Fig, 1. Sida sylvatica Cav., facsimil de la lámina original, tomada de Cavanilles 1788, Diss. 5, tab. 133, fig. 2. (Lamarck, 1794).

es rara en la familia, pero en este género hay 9 especies con frutos con más de 20 mericarpos, de las cuales 4 tienen 30 a 42 mericarpos [G.meridionalis Hass1., G.monosperma 
(K.Schum.) Krapov., G.occidentalis (L.)Sweet y G.rubricaulis Rusby].

El mericarpo dibujado por Cavanilles es parecido a los de G.albiflora Krapov, G.matutina Krapov., G.mollendoense Krapov., G.purpurea Krapov., G.scopulorum Krapov., y G.Woodii Krapov. (Krapovickas 1996, 2006, 2008).

"Semina basi acuta" también es frecuente en especies de Gaya con semillas con un extremo agudo.

El hecho de que Cavanilles haya separado la semilla para dibujarla indica que en Sida sylvatica no existe la endoglosa característica de la mayoría de sus especies. No existe en en G. monosperma (K.Scum.) Krapov. y $G$. meridensis Krapov., o bien es incipiente como en G. Guerkeana K.Schum., G. ibitipocana Krapov., G. Kelleri Krapov. y G. pilosa K.Schum. Por esta razón creo que G. sylvatica debe ubicarse en la sección Microlophia.

Gaya sylvatica (Cav.) Krapov. se diferencia del resto de las especies del género, cuyas flores son siempre solitarias, por sus flores de a dos por axila, por sus hojas grandes, las mayores del género, "longissime acuminata" y por el porte "arborescente" (Cavanilles, 1786).

\section{Gaya Kelleri Krapov., sp. nov.}

Fig. 2

Frutex usque ad 5 maltus, velutinus. Folia discolora, ovata, cordata, apice acuto, margine vix dentatoserrato. Racemus terminalis multiflorus. Calyx 6-7 mm longo, velutinus. Petala lutea, $10 \mathrm{~mm}$ longa et $16 \mathrm{~mm}$ lata. Carpidia 16-17, $6 \mathrm{~mm}$ longa et $5 \mathrm{~mm}$ lata, pilis glandularis; endoglosa $2 \mathrm{~mm}$ longa, dentata.

Typus: ARGENTINA, Misiones, Dep. San Ignacio. Estuario al norte del arroyo Chuño. $27^{\circ} 12^{\prime} 18,6^{\prime \prime} \mathrm{S} 55^{\circ} 36^{\prime} 11,5^{\prime \prime} \mathrm{W}, 152 \mathrm{~m}$. Franja de tierra rodeada de agua, pastizal. Ejemplar de $5 \mathrm{~m}$ alt. x 1,8 $\mathrm{cm}$ diám. en la base, flores amarillo-obscuras. 21II-2012. H.A.Keller \& C.J.Keller 10806 (holotypus CTES, isotypi $\mathrm{G}, \mathrm{K}, \mathrm{LIL}, \mathrm{MO}, \mathrm{NY}, \mathrm{SI})$.

Arbusto 2-4 $\mathrm{mm}$ y hasta $5 \mathrm{~m}$ alt. Tallo velutino, cubierto de pelos estrellados muy pequeños, densos. Estípulas 4-5 mm long., filiformes, caducas. Pecíolo $4 \mathrm{~cm}$ long., velutino.
Lámina ovada, hasta $6 \mathrm{~cm}$ long. x $3 \mathrm{~cm}$ lat., base cordada, ápice agudo, margen levemente cordado-aserrado, discolora, ambas caras velutinas, cubiertas de pelos estrellados muy pequeños, verde más claro en el envés. Flores en un racimo terminal alargado, plurifloro. Pedicelos 2-4 cm long., articulados 5-10 mm por debajo del cáliz, velutinos. Cáliz 6-7 mm long., velutino, liso, en fruto con tres costillas sobresalientes por lóbulo. Pétalos amarillos, $10 \mathrm{~mm}$ long. x $16 \mathrm{~mm}$ lat., uña pilosa. Tubo estaminal $4 \mathrm{~mm}$ long., glabro. Carpelos 1617. Mericarpos $6 \mathrm{~mm}$ long. x $5 \mathrm{~mm}$ lat. lisos, negruzcos, con pocos pelos glandulares en el dorso, indehicentes sólo en la base, en el interior una lengüeta basal, $2 \mathrm{~mm}$ long., con el margen dentado. Semilla $2 \mathrm{~mm}$ long., con pequeñas prominencias punctiformes. $2 n=12$ cromosomas (G. Lavia, ined.).

Distribución geográfica: Hasta el momento sólo se hallaron dos poblaciones ubicadas al norte de la desembocadura del arroyo Chuño en un amplio estuario con bañados bajos, paralelo a la orilla del río Paraná, en el departamento San Ignacio, Corrientes, Argentina.

Paratypi: ARGENTINA, Misiones, Dep. San Ignacio. $27^{\circ} 12^{\prime} 55,5^{\prime}{ }^{\prime} \mathrm{S} 55^{\circ} 35^{\prime} 39,4$ ' W, $90 \mathrm{~m}, 26-\mathrm{I}-$ 2012, H.A.Keller \& C,J.Keller 10650 (CTES, SI); Estuario al norte del arroyo Chuño . 27 12 '54,7'S 55'35'40,2”'W, 103 m, 21-II-2012, H.A.Keller \& C.J.Keller 10803 (CTES).

Obs.: Gaya Kelleri se distingue por su altura, hasta $5 \mathrm{~m}$, por las flores en racimos terminales plurifloros y por su endoglosa de $2 \mathrm{~mm}$ long., dentada, caracteres que la aislan en el género. Por la presencia de una endoglosa diminuta, de $2 \mathrm{~mm}$ long. en sus mericarpos Gaya Kelleri se ubica en la sección Microlophia K. Schum.

Etimología: Especie dedicada a Hector A. Keller, quién coleccionó el material estudiado.

\section{Gaya pilosa K.Schum.}

Schumann, K. en Mart. Fl. bras. 12(3): 355-356, tab. 64, fig. 1, 1891. 


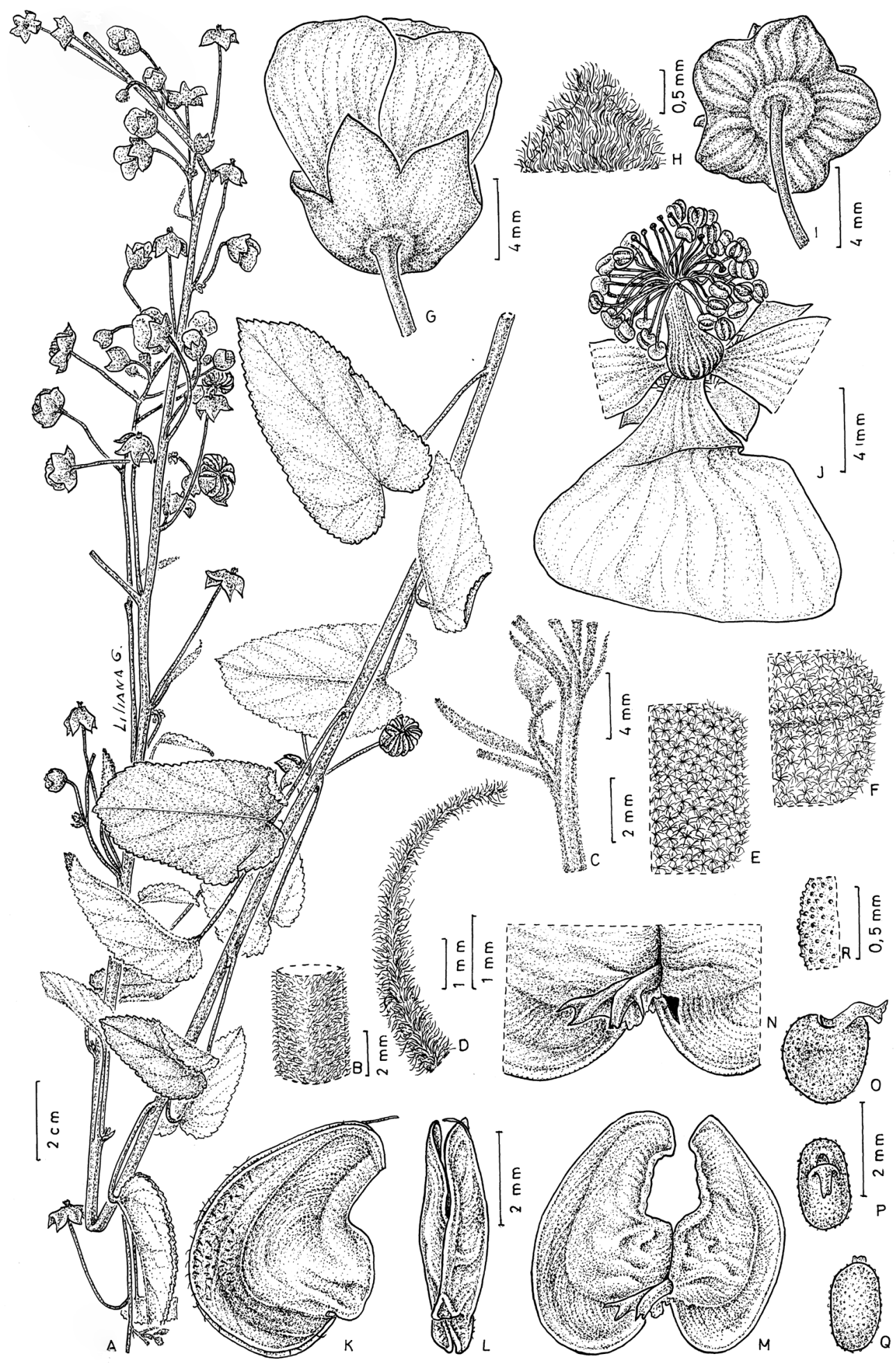

Fig. 2. Gaya Kelleri. A: rama. B: indumento del tallo. C: estípulas. D: indumento de las estípulas. E: indumento del epifilo. F: indumento del hipofilo. G: cáliz. H: indumento del cáliz. I: relieve del cáliz en el fruto. J: pétalo y tubo estaminal. K: mericarpo, vista lateral. L: mericarpo vista ventral. M: mericarpo abierto. N: endoglosa del mericarpo. O, P, Q: semillas. R: superficie de la semilla (Keller \& Keller 10806). 
Gaya ibitipocana Krapov. Bonplandia 17(1): 3738, fig. 2. 2008. Holotypus: Brasil, Minas Gerais, Lima Duarte, Serra do Ibitipoca, Forzza 4720 (RB) (sinónimo nuevo).

Al analizar nuevamente el material de Gaya, encuentro que el ejemplar Forzza 4720, tipo de G.ibitipobana Krapov. no se puede separar de los ejemplares de G.pilosa, por lo cual considero a Gaya ibitipocana Krapov. como sinónimo de G.pilosa K.Schum.

\section{Bibliografía}

CANDOLLE, A.P. de. 1824. Malvaceae in Prodomus systematis naturalis regni vegetabilis. ed. A.P. de Candolle 1: 427-474. Paris. Trettel \& Würtz.

CAVANILLES, A.J. 1786. Secunda Dissertatio botanica. Franciscum Amb. Didot, Paris.

—. 1788. Quinta Dissertatio botanica. Franciscum Aub. Didot, Paris.

DON, G. 1831-1835. General history of the dichlamydeous plants. 4 vols. London. [Malvaceae en vol.1: 458$505]$.
FRYXELL,P.A. 1993. Malvaceae. In G.Harling \& L.Andersson (eds.) Flora of Ecuador 44.

- 2002. An Abutilon nomenclator (Malvaceae). Lundellia 5:79-118.

FUERTES, J. and P.A.FRYXELL. 1993. Nomenclatural notes on some Malvaceae species described by Cavanilles. Taxon 42: 661-664.

GARCKE, A. 1893. Ueber die Gattung Abutilon. Bot. Jahrb. Syst. 15: 480-492.

HANKS, S. \& P.A. FRYXELL. 1979. Palynological studies of Gaya and Herissantia (Malvaceae). Amer. J. Bot. 66(5): 494-501.

KRAPOVICKAS, A. 1996. Sinopsis del género Gaya (Malvaceae). Bonplandia 9 (1-2): 57-87.

- 2006. Novedades sudamericanas en Acaulmalva y Gaya (Malvaceae-Malveae). Bonplandia 15(3-4): 103-112.

_. 2008. Nuevas especies de Malvaceae. Bonplandia 17(1): 35-45.

- S.G. TRESSENS \& A. FERNÁNDEZ, 1974. Gaya elingulata, nueva especie de Malvácea de Brasil. Bol. Soc. Argent. Bot. 16(1-2): 93-96.

LAMARCK, J.B.A.M. de. 1794. Tableau encyclopedique et metodique des trois rêgnes de la nature. Botanique. 3, tab. 578

PRESL, K. 1830-1835. Reliquiae haenkeanae. Prague. 2 vols.

Original recibido el 24 de abril de 2012; aceptado el 19 de junio de 2012. 